\title{
ASYMPTOTICS OF REPRODUCING KERNELS ON A PLANE DOMAIN
}

\author{
MIROSLAV ENGLIŠ \\ (Communicated by Theodore W. Gamelin)
}

\begin{abstract}
Let $\Omega$ be a plane domain of hyperbolic type, $|d z| / w(z)$ the Poincare metric on $\Omega$, and $K_{\Omega, q}(x, \bar{y})$ the reproducing kernel for the Hilbert space $\mathscr{A}_{q}^{2}(\Omega)$ of all holomorphic functions on $\Omega$ square-integrable with respect to the measure $w(z)^{2 q-2}|d z \wedge d \bar{z}|$. It is proved that
\end{abstract}

$$
\lim _{q \rightarrow+\infty} \frac{K_{\Omega, q}(z, \bar{z}) w(z)^{2 q}}{2 q}=\frac{1}{\pi} \text {. }
$$

Let $\Omega \subset \mathbf{C}$ be a domain of hyperbolic type (i.e., $\mathbf{C} \backslash \Omega$ contains at least two points), so that the universal covering surface of $\Omega$ is isomorphic to the unit disc $\mathbf{D}$. Let $\phi: \mathbf{D} \rightarrow \Omega$ be the covering map and

$$
G=\{\omega \in \operatorname{Aut}(\mathbf{D}): \phi \circ \omega=\phi\}
$$

the corresponding group of covering transformations. $G$ acts freely and properly discontinuously on $\mathbf{D}$, and $\Omega$ may be identified with the coset space $\mathbf{D} / G$. The Poincaré metric on $\Omega$, corresponding to the hyperbolic metric $\frac{|d z|}{1-|z|^{2}}$ on $\mathbf{D}$, is given by $d s=\frac{|d x|}{w(x)}$, where (see [1, section II.1])

$$
w(\phi(z))=\left(1-|z|^{2}\right)\left|\phi^{\prime}(z)\right| \text {. }
$$

The identity

$$
1-|\omega(z)|^{2}=\left(1-|z|^{2}\right)\left|\omega^{\prime}(z)\right|, \quad z \in \mathbf{D}, \omega \in \operatorname{Aut}(\mathbf{D}),
$$

which can be verified by a short computation, shows that the right-hand side indeed depends only on $\phi(z)$, so that the definition of $w(x)$ is correct.

The Bergman space $A_{\alpha}^{2}(\Omega)$ consists, by definition, of all holomorphic functions on $\Omega$ square-integrable against the measure $w(x)^{\alpha} d E(x)$, where $d E$ is the Lebesgue area measure. We shall only be interested in the case when $\alpha$ is an even integer: $\alpha=2 q-2(q \in \mathbf{Z})$, and we will write $\mathscr{A}_{q}^{2}(\Omega)$ instead of $A_{\alpha}^{2}(\Omega)$ in that case. Endowed with the Petersson scalar product

$$
\langle f, g\rangle_{q}=\int_{\Omega} f(x) \overline{g(x)} w(x)^{2 q-2} d E(x),
$$

Received by the editors March 30, 1994.

1991 Mathematics Subject Classification. Primary 30C40, 30E15.

Key words and phrases. Bergman kernel, automorphic functions. 
$\mathscr{A}_{q}^{2}(\Omega)$ becomes a Hilbert space. For $q \geq 2$ (or, if $\Omega \notin O_{G}$, for $q \geq 1$ ), it can be shown that $\mathscr{A}_{q}^{2}(\Omega) \neq\{0\}$ and that $\mathscr{A}_{q}^{2}(\Omega)$ admits a reproducing kernel (see [1, Chapter III]): there exists a function $K_{\Omega, q}(x, \bar{y}) \quad(x, y \in \Omega)$, holomorphic in $x$ and antiholomorphic in $y$, such that

$$
\begin{aligned}
& f(x)=\left\langle f, K_{\Omega, q}(\cdot, \bar{x})\right\rangle_{q}=\int_{\Omega} f(y) K_{\Omega, q}(x, \bar{y}) w(x)^{2 q-2} d E(x), \\
& \forall f \in \mathscr{A}_{q}^{2}(\Omega), x \in \Omega .
\end{aligned}
$$

Our main goal is to prove the following result.

Theorem. For any $x \in \Omega$,

$$
\lim _{q \rightarrow+\infty} \frac{K_{\Omega, q}(x, \bar{x}) w(x)^{2 q}}{2 q}=\frac{1}{\pi} .
$$

For $\Omega=\mathbf{D}$, this is trivial, and for $\Omega$ an annulus or a punctured disc, this has been obtained (by a totally different method) in [3] and applied to the study of the asymptotics of the Berezin transform. The latter, in turn, provides the basic tool for certain quantization procedures on $\Omega$ (construction of *-products) [4], [5].

Proof. We will borrow the notation and some results from Chapter III of Kra [1]. Let $\Delta \subset \mathbf{D}$ be a fundamental domain for $G$ (see Tsuji [2, section XI.2]). Denote

$$
\begin{aligned}
\mathscr{O}(\mathbf{D})= & \{\text { holomorphic functions on } \mathbf{D}\}, \\
\|f\|_{q, G}= & \left(\int_{\Delta}|f(z)|^{2}\left(1-|z|^{2}\right)^{2 q-2} d E(z)\right)^{1 / 2}, \\
\mathscr{A}_{q}^{2}(\mathbf{D}, G)= & \left\{f \in \mathcal{O}(\mathbf{D}):\|f\|_{q, G}<+\infty\right. \\
& \text { and } \left.f(z)=f(\omega(z)) \cdot \omega^{\prime}(z)^{q} \quad \forall \omega \in G\right\} .
\end{aligned}
$$

The mapping

$$
f(x) \mapsto f(\phi(z)) \phi^{\prime}(z)^{q}, \quad x \in \Omega, z \in \mathbf{D},
$$

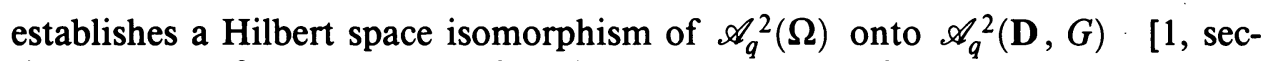
tion III.6]. It follows that the function $F_{q, G}(z, \bar{y})$, defined by

$$
F_{q, G}(z, \bar{y})=K_{\Omega, q}(\phi(z), \overline{\phi(y)}) \cdot \phi^{\prime}(z)^{q} \overline{\phi^{\prime}(y)^{q}}, \quad z, y \in \mathbf{D},
$$

is the reproducing kernel for the Hilbert space $\mathscr{A}_{q}^{2}(\mathbf{D}, G)$. But, according to section III.5 of [1], the latter reproducing kernel is also given by the Poincare series

$$
F_{q, G}(z, \bar{y})=\sum_{\omega \in G} K_{\mathbf{D}, q}(\omega(z), \bar{y}) \omega^{\prime}(z)^{q}, \quad z, y \in \mathbf{D},
$$

where $K_{\mathbf{D}, q}$ is the reproducing kernel for the Bergman space $\mathscr{S}_{q}^{2}(\mathbf{D})$ on the unit disc D. Summarizing, we have

$$
\begin{aligned}
\lim _{q \rightarrow+\infty} \frac{K_{\Omega, q}(x, \bar{x}) w(x)^{2 q}}{2 q} & =\lim _{q \rightarrow+\infty} \frac{F_{q, G}(z, \bar{z})\left|\phi^{\prime}(z)\right|^{-2 q} \cdot\left(1-|z|^{2}\right)^{2 q}\left|\phi^{\prime}(z)\right|^{2 q}}{2 q} \\
& =\lim _{q \rightarrow+\infty} \frac{\left(1-|z|^{2}\right)^{2 q}}{2 q} \sum_{\omega \in G} K_{\mathbf{D}, q}(\omega(z), \bar{z}) \omega^{\prime}(z)^{q},
\end{aligned}
$$


where $x=\phi(z) \quad(x \in \Omega, z \in \mathbf{D})$. Now it is well known that

$$
K_{\mathbf{D}, q}(y, \bar{z})=\frac{2 q-1}{\pi}(1-\bar{z} y)^{-2 q} \text {. }
$$

Therefore

$$
\begin{aligned}
& \lim _{q \rightarrow+\infty} \frac{\left(1-|z|^{2}\right)^{2 q}}{2 q} \sum_{\omega \in G} K_{\mathbf{D}, q}(\omega(z), \bar{z}) \omega^{\prime}(z)^{q} \\
&=\frac{1}{\pi} \lim _{q \rightarrow+\infty} \sum_{\omega \in G}\left[\left(1-|z|^{2}\right)^{2}(1-\bar{z} \omega(z))^{-2} \omega^{\prime}(z)\right]^{q} .
\end{aligned}
$$

For $a \in \mathbf{D}$, let $\omega_{a} \in \operatorname{Aut}(\mathbf{D})$ be the Möbius transformation given by

$$
\omega_{a}(y)=\frac{y-a}{1-\bar{a} y}
$$

Then

It follows that

$$
\omega_{a}^{\prime}(y)=\frac{1-|a|^{2}}{(1-\bar{a} y)^{2}}, \quad \omega_{a}^{\prime}(0)=1-|a|^{2} .
$$

$$
\begin{aligned}
\left(1-|z|^{2}\right)^{2}(1-\bar{z} \omega(z))^{-2} \omega^{\prime}(z) & =\left(1-|z|^{2}\right) \cdot \omega_{z}{ }^{\prime}(\omega(z)) \cdot \omega^{\prime}(z) \\
& =\omega_{-z}{ }^{\prime}(0) \cdot \omega_{z}{ }^{\prime}(\omega(z)) \cdot \omega^{\prime}(z) \\
& =\left(\omega_{z} \omega \omega_{-z}\right)^{\prime}(0) .
\end{aligned}
$$

Let $\lambda=\omega_{z} \omega \omega_{-z} \in \operatorname{Aut}(\mathbf{D})$. By the Schwarz lemma (or direct computation),

$$
\left|\lambda^{\prime}(0)\right|=1-|\lambda(0)|^{2} \leq 1,
$$

with equality occurring iff $\lambda(0)=0$, i.e. iff $\omega(z)=z$. Since $G$ acts freely, this is only possible for $\omega=\mathrm{id}$. Thus

$$
\left|\left(\omega_{z} \omega \omega_{-z}\right)^{\prime}(0)\right|<1 \quad \text { for } \omega \in G \backslash\{\text { id }\} .
$$

Let us now make the following elementary observation: whenever $\left\{b_{\nu}\right\}_{\nu \in I}$ is $a$ sequence of complex numbers, indexed by a countable set $I$, such that

(a) $\sum_{\nu \in I}\left|b_{\nu}\right|^{q}<+\infty$ for some $q>0$, and

(b) $\left|b_{\nu}\right|<1$ for all $\nu$,

then $\sum_{\nu \in I} b_{\nu}^{q} \rightarrow 0$ as $q \rightarrow+\infty$.

Indeed, it follows from (a) that for any $\epsilon>0$ there is at most a finite number of $b_{\nu}$ with $\left|b_{\nu}\right|>\epsilon$; so there exists $\nu_{0} \in I$ such that $\left|b_{\nu_{0}}\right|=\sup _{I}\left|b_{\nu}\right|$. Then

$$
\left|\sum_{I} b_{\nu}^{q}\right| \leq\left|b_{\nu_{0}}^{q}\right| \sum_{I}\left|b_{\nu} / b_{\nu_{0}}\right|^{q}
$$

and as $\left|b_{\nu} / b_{\nu_{0}}\right| \leq 1$ for all $\nu$, the last sum is a nonincreasing function of $q$. It follows that $\sum_{I} b_{\nu}^{q}=O\left(\left|b_{\nu_{0}}\right|^{q}\right)$, and as $\left|b_{\nu_{0}}\right|<1$ by (b), the assertion follows.

Since

$$
\begin{aligned}
\sum_{\omega \in G}\left|\left(\omega_{z} \omega \omega_{-z}\right)^{\prime}(0)\right|^{2} & =\sum_{\omega \in G}\left(1-\left|\omega_{z} \omega(z)\right|^{2}\right)^{2}=\sum_{\omega \in G}\left[\frac{\left(1-|z|^{2}\right)\left(1-|\omega(z)|^{2}\right)}{|1-\bar{z} \omega(z)|^{2}}\right]^{2} \\
& \leq\left(\frac{1+|z|}{1-|z|}\right)^{2} \sum_{\omega \in G}\left(1-|\omega(z)|^{2}\right)^{2}
\end{aligned}
$$


is finite by [1, Lemma III.5.2], the above observation can be applied to $I=$ $G \backslash\{\mathrm{id}\}, b_{\omega}=\left(\omega_{z} \omega \omega_{-z}\right)^{\prime}(0)$, and shows that

$$
\sum_{\omega \in G \backslash\{\mathrm{id}\}}\left[\left(\omega_{z} \omega \omega_{-z}\right)^{\prime}(0)\right]^{q} \rightarrow 0 \quad \text { as } q \rightarrow+\infty .
$$

Consequently,

$$
\begin{aligned}
\lim _{q \rightarrow+\infty} \sum_{\omega \in G}\left[\left(1-|z|^{2}\right)^{2}(1-\bar{z} \omega(z))^{-2} \omega^{\prime}(z)\right]^{q} & =\lim _{q \rightarrow+\infty} \sum_{\omega \in G}\left[\left(\omega_{z} \omega \omega_{-z}\right)^{\prime}(0)\right]^{q} \\
& =\lim _{q \rightarrow+\infty}\left[\left(\omega_{z} \cdot \mathrm{id} \cdot \omega_{-z}\right)^{\prime}(0)\right]^{q} \\
& =1
\end{aligned}
$$

and the proof is finished.

Problem. In view of the result just proved, the following question now seems to be of some interest. Let $F$ be a positive continuous function on the interval $[0,1)$ and let $K_{\alpha}$ be the reproducing kernel for the Bergman space $A^{2}\left(\mathbf{D}, F\left(|z|^{2}\right)^{\alpha} d E(z)\right)$. For which $F$ do the functions

$$
K_{\alpha}(z, \bar{z}) F\left(|z|^{2}\right)^{\alpha} / \alpha
$$

converge as $\alpha \rightarrow+\infty$, and if they do, what is the limit?

\section{REFERENCES}

1. I. Kra, Automorphic forms and Kleinian groups, Benjamin, Reading, MA, 1972.

2. M. Tsuji, Potential theory in modern function theory, Maruzen, Tokyo, 1959.

3. M. Engliš and J. Peetre, On the correspondence principle for the quantized annulus, Math. Scand. (to appear).

4. F.A. Berezin, General concept of quantization, Comm. Math. Phys. 40 (1975), 153-174.

5. M. Cahen, S. Gutt, and J. Rawnsley, Quantization on Kähler manifolds II, Trans. Amer. Math. Soc. 337 (1993), 73-98.

MÚ AV Čr, Žitná 25, 11567 Prague 1, Czech Republic

E-mail address: englis@cspgas11.bitnet, englis@earn.cvut.cz 